\title{
Research Paper \\ Comparing the Effect of SubMucosal Resection With Electrocautery Method for the Treatment of Inferior Turbinate Hypertrophy at Amir Kabir Hospital in Arak City
}

\section{*Farzad Zamani Barsari ${ }^{1}$ (1)}

1. Department of ENT, School of Medicine, Arak University of Medical Science, Arak, Iran.

\begin{tabular}{c|l}
$\begin{array}{c}\text { Use your device to scan } \\
\text { and read the article online }\end{array}$ \\
$\begin{array}{l}\text { Cftation: Zamani Barsari F. [Comparing the Effect of Submucosal Resection With Electrocautery Method for the Treatment } \\
\text { of Inferior Turbinate Hypertrophy at Amir Kabir Hospital in Arak City (Persian)]. Journal of Arak University of Medical Sciences } \\
\text { (JAMS). 2019; 22(4):66-75. https://doi.org/10.32598/JAMS.22.4.66 }\end{array}$ \\
doisths://doi.org/10.32598/JAMS.22.4.66
\end{tabular}

\section{(i) $(8)$}

Article Info:

Received: 11 Jun 2018

Accepted: 07 Mar 2019

Available Online: 01 Oct 2019
Key words:

Inferior turbinate hypertrophy, Electrocautery, SubMucosal Resection (SMR)

\section{A B S TRACT}

Background and Aim Inferior turbinate hypertrophy is one of the most common causes of nasal obstruction after nasal septal deviation. Enlargement of the turbinate is the result of the position of turbinate bone or swelling of turbinate submucosal. This study aimed to evaluate the effectiveness of the SubMucosal Resection (SMR) method with electrocautery method in the treatment of inferior turbinate hypertrophy.

Methods and Materials A total of 140 patients with inferior turbinate hypertrophy complicated with nasal congestion and rhinorrhea and candidate of surgery were randomly divided into two groups. In the first group, after induction of anesthesia, we performed SMR of inferior turbinate hypertrophy. In the second group, after induction of anesthesia, we performed monopolar cauterization with 20-gauge angiocath in three anterior, middle, and posterior regions and crests of the inferior turbinate.

Ethical Considerations This study was approved by the Ethics Committee of Arak University of Medical Sciences (Code: 91.133.3).

Results In the electrocautery group, a significant improvement was observed in nasal congestion after 6 months $(P=0.017)$. In the SMR group, a satisfactory headache improvement was reported $(P=0.034)$, one month after the operation. Also, the electrocautery group reported less pain and burning in the site of surgery, sneezing, coughing, and itching $(\mathrm{P}=0.013)$.

Conclusion Electrocautery can be superior over SMR because of its less bleeding, shorter time of surgery, the availability of primary equipment, and relative improvement in decreasing the rhinorrhea. However, if the patient suffers from isolated inferior turbinate hypertrophy, SMR as a suitable method is recommended.

\section{Extended Abstract}

\section{Introduction}

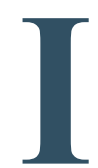

nferior Turbinate Hypertrophy (ITH) is one of the most common causes of nasal obstruction after nasal deviation, which includes mucosal, submucosal, and bone hypertrophy. There are various types of medications and operations for the treatment of ITH and can be performed depending on the availability of the equipment, budget, complications, and skill of the surgeon. One of the primary treatment methods is radiofrequency. However, it is an expensive procedure, and its equipment is not available everywhere. Thus, the researchers try to find and replace it with cost-effective and convenient methods. This study aimed to compare the effectiveness of SubMu-

\section{* Corresponding Author:}

Farzad Zamani Barsari, PhD.

Address: Department of ENT, School of Medicine, Arak University of Medical Science, Arak, Iran.

Tel: +98 (918) 7575839

E-mail: farzadzamani1393@gmail.com 
cosal Resection (SMR) with the electrocautery method in the treatment of ITH.

\section{Materials and Methods}

In this study, 140 patients with ITH who complained of nasal congestion and rhinorrhea were selected for the study. Their nasal obstruction was evident, according to rhinoscopy. Also, they had not responded to drug therapy, such as oral or topical corticosteroids, antihistamines, and other conservative methods. Thus, they were candidates for surgery. In the next step, they were divided into two groups of 70 patients by simple random method. In the first group, after induction of anesthesia, SMR was performed, and the hypertrophic tissue was removed by incision method. In the second group, monopolar cauterization was performed in anterior, medial, and posterior areas of inferior turbinate after induction of anesthesia. Then, during the first week and later in the first, third, and sixth months after surgery the information on nasal congestion, Post-Nasal Drip (PND), rhinorrhea, snoring, headache, sneezing, itchy throat, hyposmia, anosmia, nose bleeding, crust, and severity of these symptoms was recorded in a questionnaire.

\section{Results}

Tables 1, 2 and 3 presents the prevalence of headache, rhinorrhea, and nose bleeding in the two groups.

\section{Discussion}

The results of this study showed that patients' complaints of headache, nasal congestion, rhinorrhea, nose bleeding, itching, sneezing, and crust in both groups were different at different time intervals after surgery. However, since the electrocautery method had less bleeding and took less surgical time, had available primary equipment and caused relatively reduced PND and rhinorrhea, this method seems to have a relative advantage over the SMR method. Thus, if the patient has isolated submucosal and mucosal hypertrophy of inferior turbinate (bone of the inferior turbinate is not involved), it could be recommended as the preferred method.

\section{Ethical Considerations}

\section{Compliance with ethical guidelines}

This study was approved by the Research Ethics Committee of Arak University of Medical Sciences (Code: 91.133.3). All ethical principles were met in this study. The

Table 1. Prevalence of headache after surgery in two study groups

\begin{tabular}{|c|c|c|c|c|c|}
\hline & \multirow{2}{*}{ Headache } & \multicolumn{2}{|c|}{ Group } & \multirow{2}{*}{ Total } & \multirow{2}{*}{$\mathbf{P}$} \\
\hline & & SMR & Electrocautery & & \\
\hline \multirow{4}{*}{ First week } & No change & 24 & 21 & 45 & \multirow{4}{*}{0.923} \\
\hline & Deterioration & 44 & 46 & 90 & \\
\hline & Relative recovery & 2 & 3 & 5 & \\
\hline & Satisfactory recovery & 0 & 0 & 0 & \\
\hline \multirow{4}{*}{ One month later } & No change & 49 & 51 & 100 & \multirow{4}{*}{0.034} \\
\hline & Deterioration & 0 & 3 & 3 & \\
\hline & Relative recovery & 14 & 16 & 30 & \\
\hline & Satisfactory recovery & 7 & 0 & 7 & \\
\hline \multirow{3}{*}{ Three months later } & No change & 44 & 44 & 88 & \multirow{3}{*}{0.922} \\
\hline & Relative recovery & 19 & 21 & 40 & \\
\hline & Satisfactory recovery & 7 & 5 & 12 & \\
\hline \multirow{3}{*}{ Six months later } & No change & 44 & 43 & 87 & \multirow{3}{*}{0.157} \\
\hline & Relative recovery & 14 & 22 & 36 & \\
\hline & Satisfactory recovery & 12 & 5 & 17 & \\
\hline
\end{tabular}


Table 2. Prevalence of rhinorrhea after surgery in two study groups

\begin{tabular}{|c|c|c|c|c|c|}
\hline \multirow{2}{*}{\multicolumn{2}{|c|}{ Rhinorrhea }} & \multicolumn{2}{|c|}{ Group } & \multirow[b]{2}{*}{ Total } & \multirow{2}{*}{$\mathbf{P}$} \\
\hline & & SMR & $\begin{array}{l}\text { Electrocau- } \\
\text { tery }\end{array}$ & & \\
\hline \multirow{4}{*}{ First week } & No change & 22 & 15 & 37 & \multirow{4}{*}{0.179} \\
\hline & Deterioration & 24 & 34 & 58 & \\
\hline & Relative recovery & 24 & 21 & 45 & \\
\hline & Satisfactory recovery & 0 & 0 & 0 & \\
\hline \multirow{4}{*}{ One month later } & No change & 15 & 13 & 28 & \multirow{4}{*}{0.104} \\
\hline & Deterioration & 0 & 7 & 7 & \\
\hline & Relative recovery & 40 & 38 & 78 & \\
\hline & Satisfactory recovery & 15 & 12 & 27 & \\
\hline \multirow{3}{*}{ Three months later } & No change & 15 & 8 & 23 & \multirow{3}{*}{0.394} \\
\hline & Relative recovery & 38 & 40 & 78 & \\
\hline & Satisfactory recovery & 17 & 22 & 39 & \\
\hline \multirow{3}{*}{ Six months later } & No change & 15 & 7 & 22 & \multirow{3}{*}{0.173} \\
\hline & Relative recovery & 38 & 37 & 75 & \\
\hline & Satisfactory recovery & 17 & 26 & 43 & \\
\hline
\end{tabular}

Table 3. Prevalence of nose bleeding after surgery in two study groups

\begin{tabular}{|c|c|c|c|c|c|}
\hline \multirow{2}{*}{\multicolumn{2}{|c|}{ Nose bleeding }} & \multicolumn{2}{|c|}{ Group } & \multirow{2}{*}{ Total } & \multirow{2}{*}{$\mathbf{P}$} \\
\hline & & SMR & Electrocautery & & \\
\hline \multirow{3}{*}{ First week } & High & 6 & 0 & 63 & \multirow{3}{*}{0.001} \\
\hline & Moderate & 25 & 13 & 38 & \\
\hline & Mild & 39 & 57 & 39 & \\
\hline \multirow{3}{*}{ One month later } & No bleeding & 51 & 67 & 118 & \multirow{3}{*}{0.00} \\
\hline & & & & & \\
\hline & Mild & 19 & 3 & 22 & \\
\hline \multirow{3}{*}{ Three months later } & No bleeding & 69 & 68 & 137 & \multirow{3}{*}{1} \\
\hline & & & & & \\
\hline & Mild & 1 & 2 & 3 & \\
\hline
\end{tabular}

participants were informed about the research objective and method. They were also assured of the confidentiality of their information and were allowed to leave the study at any time, and if desired, the results of the research would be available to them.

\section{Conflicts of interest}

The author declares no conflict of interest.

\section{Funding}

This research did not receive any specific grant from funding agencies in the public, commercial, or not-forprofit sectors. 


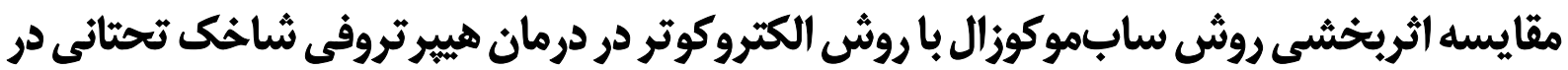

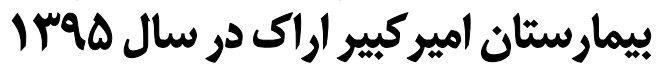

(1) 'فرزاد زمانى برسرى"

1- كروه كوش و حلق و بينى، دانشكده يزشكى، دانشكاه علوم يزشكى اراك، اراك، ايران.

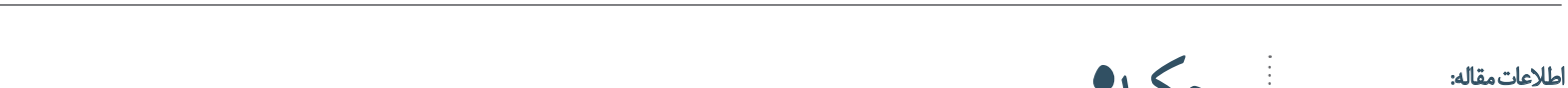

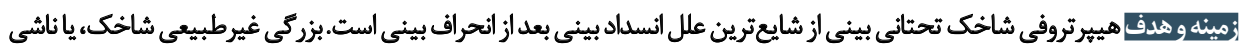

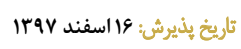

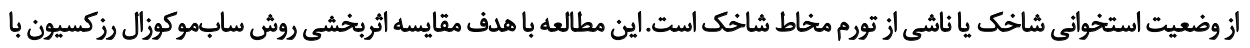
تاريخ انتشار: 9. مهر 1491

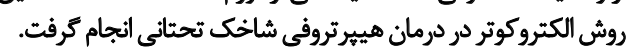

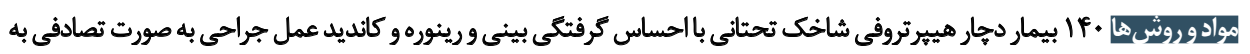

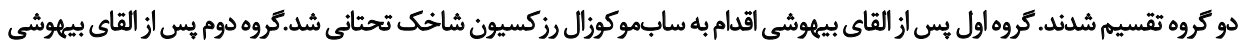

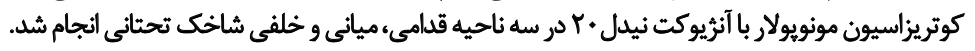

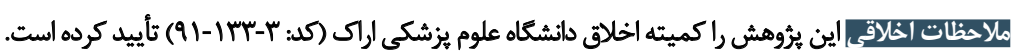

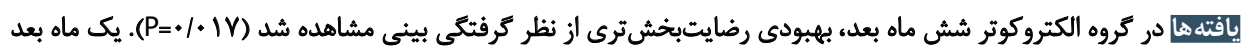

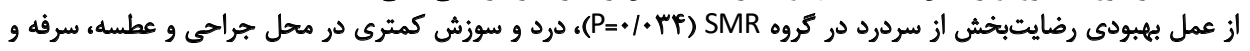

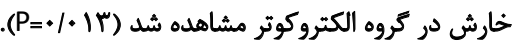

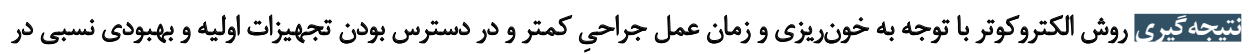

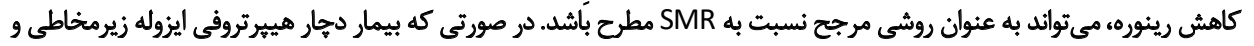

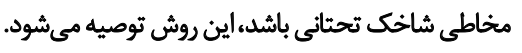

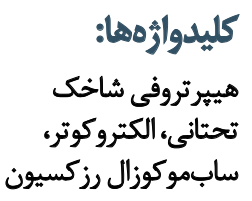

راديوفركونسى، ليزر، كرايو، سابموكوزال رزكشن ' و تزريق ثارشيال

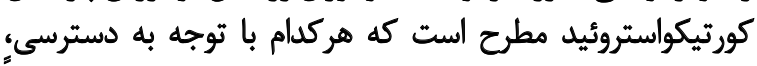

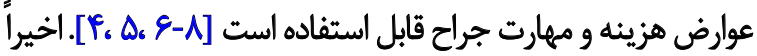

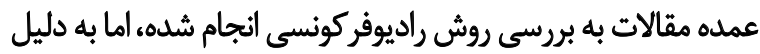

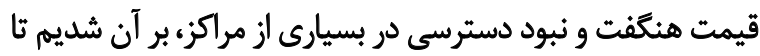

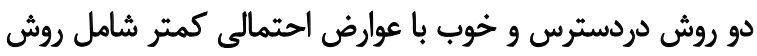

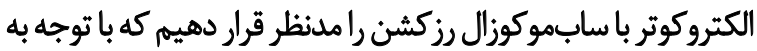

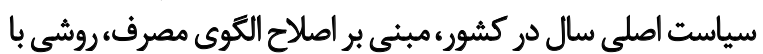

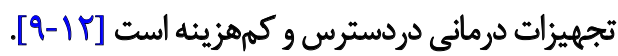
بينى فقط معبرى براى عبور هوا و رسيدن آن به به ريه ديه نيست

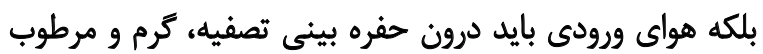

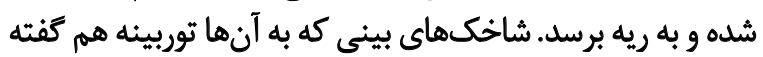

1. Submocusal Resection

dateo

هيبرتروفى شاخك تحتانى بينى از شايعترين علل انسداد بينى بئى بئي

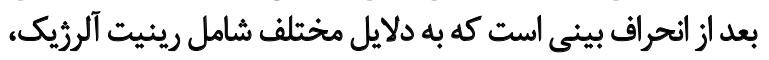

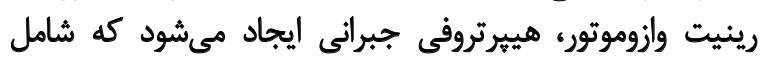

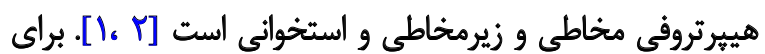

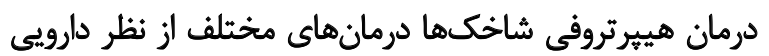

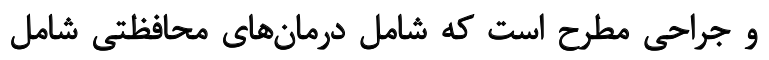

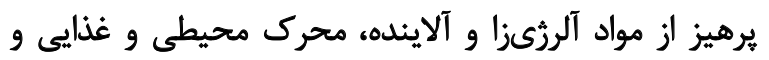

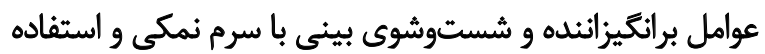

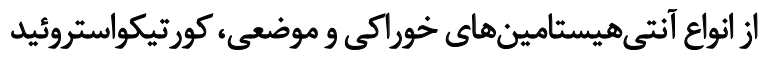

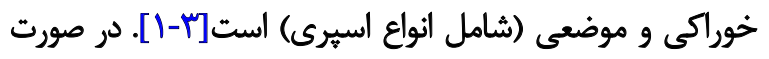

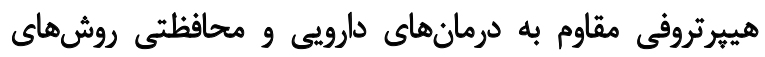
جراحى مدنظر قرار مي كيرد كه شامل الكتروكوتومونويولار ب، باى يولار، 


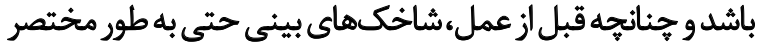

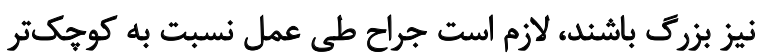

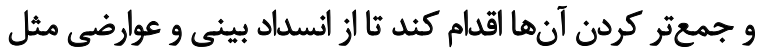

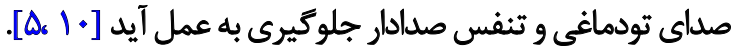

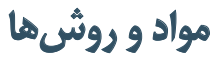

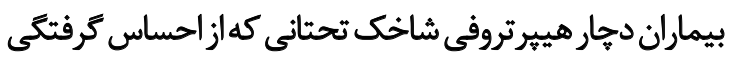

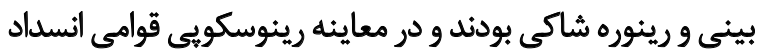

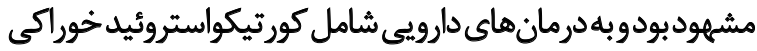

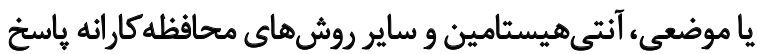

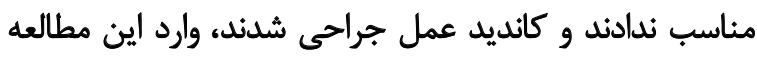

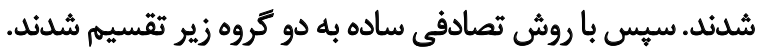

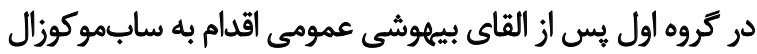

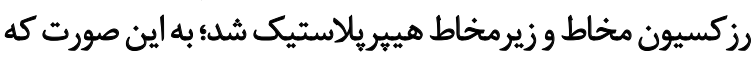

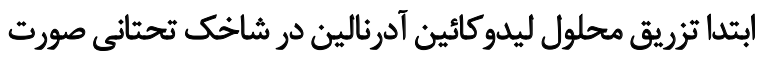

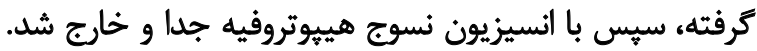

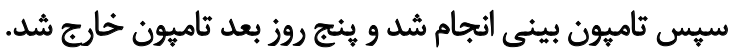

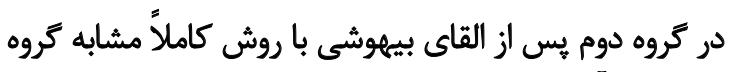

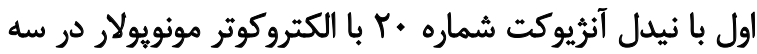

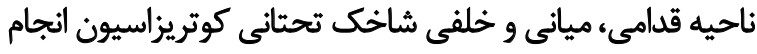

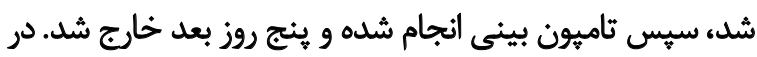

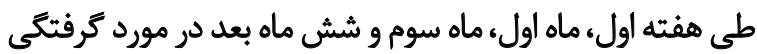

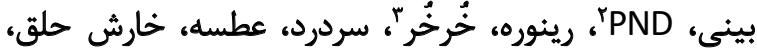

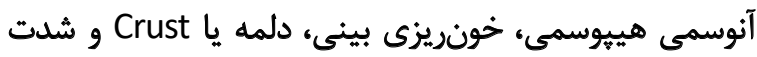

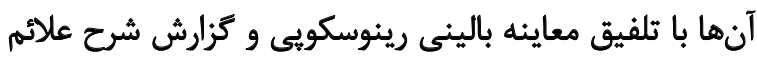

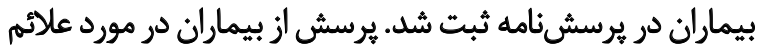

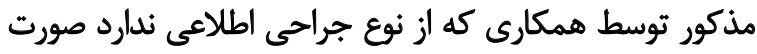

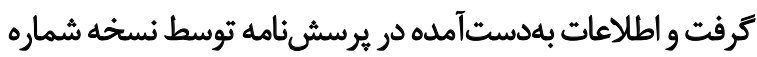
بr نرمافزار آمارى SPSS تجزيه و تحليل شد.

معيارهاى ورود شامل بيماران دجار هييرتروفى شاخك تحتانى

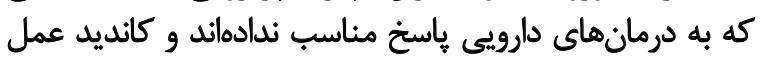

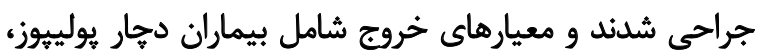
سينوزيت و انحراف سيتوم بودي.

ياقتهها

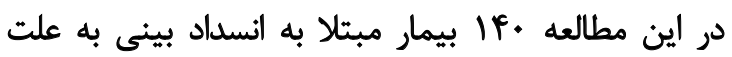

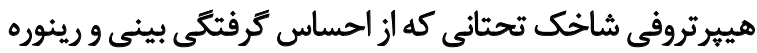

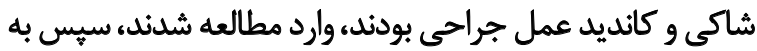

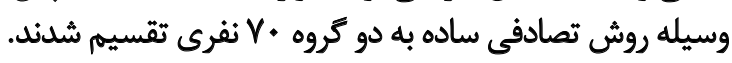

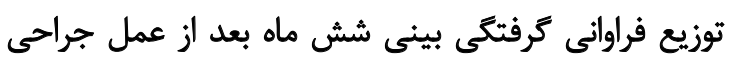

2. Post-Nasal Drip

3. Snoring
مىشود، نقش بسزايى در گرم و مرطوبكردن هواى تنفسى دارند.

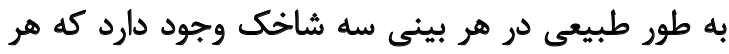

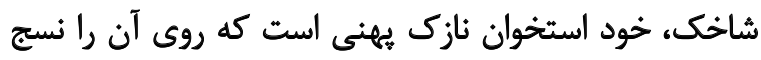

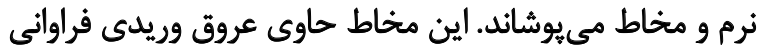

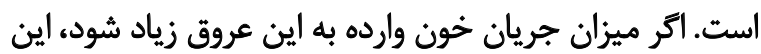

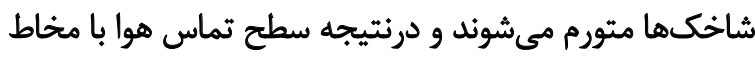

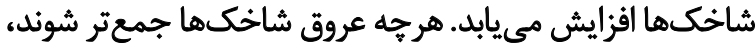

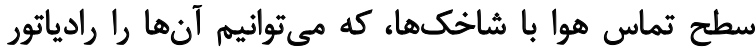

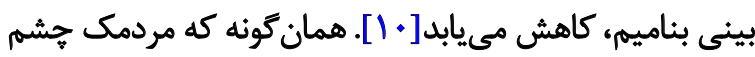

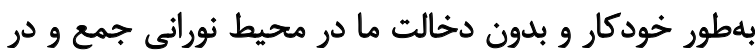

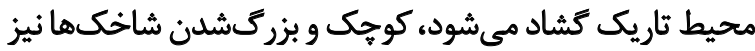

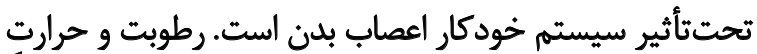

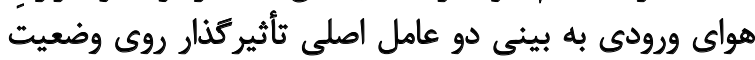

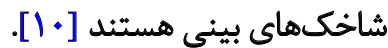

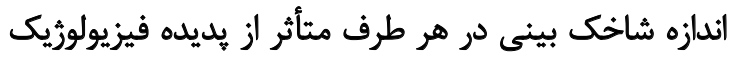

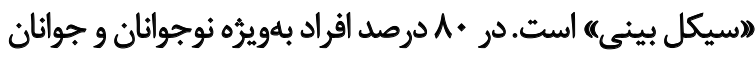

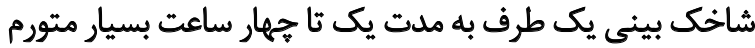

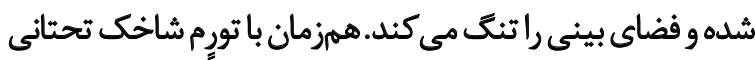

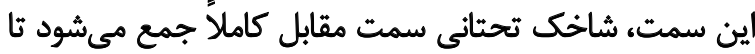

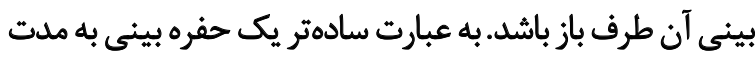

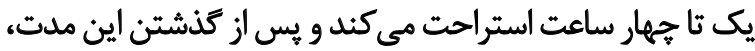

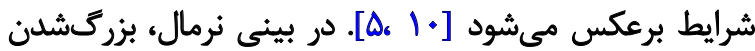

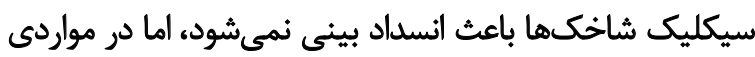

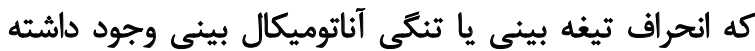

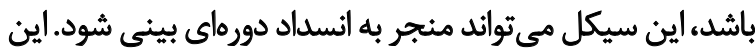

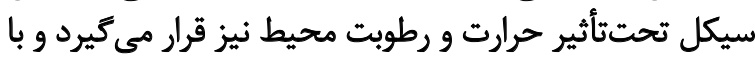

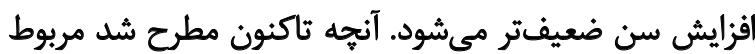

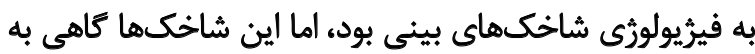

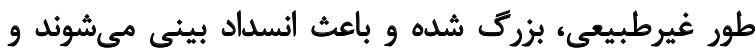

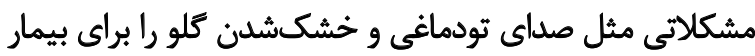

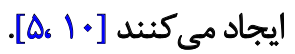

بزركى غيرطبيعى شاخك، يا ناشى از وضعيت استخوانى

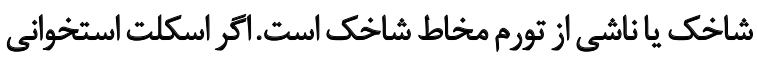

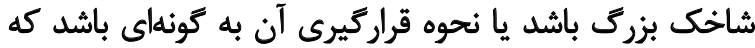

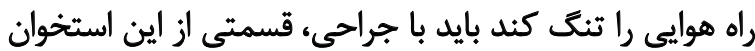

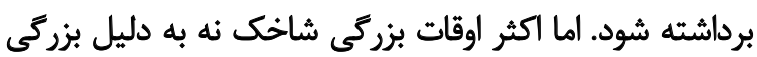

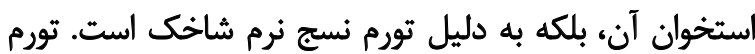

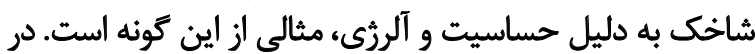

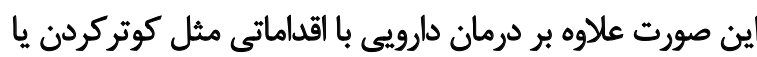

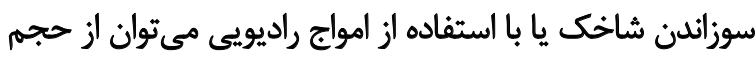

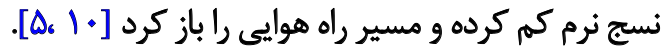

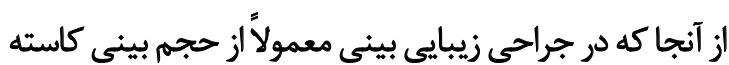
مىشود، جراح بينى بايد توجه ويرٔائى به فضاي داخل داخل بينى داشته بينه 


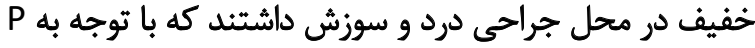

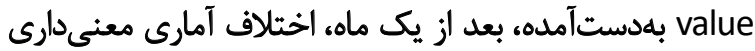

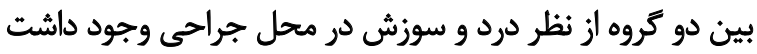

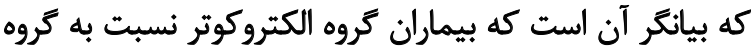

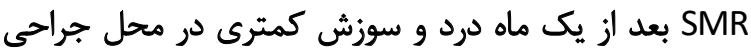

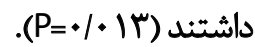

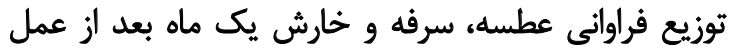

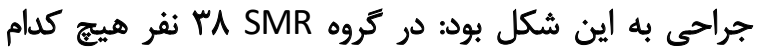

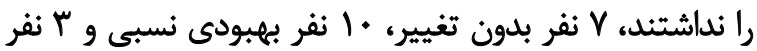

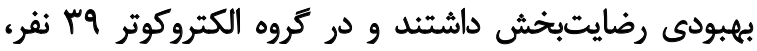

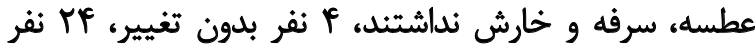

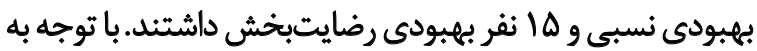

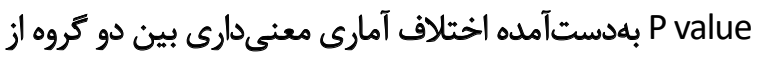

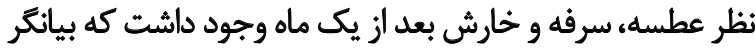

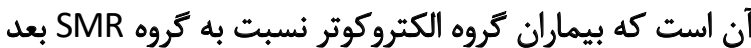

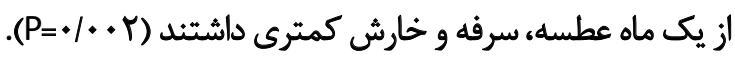

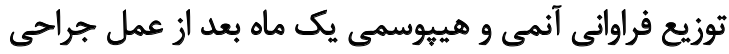

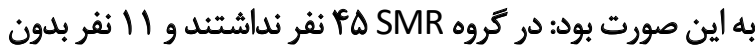

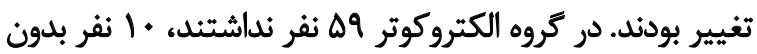

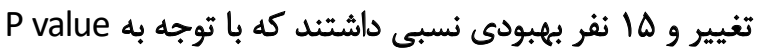

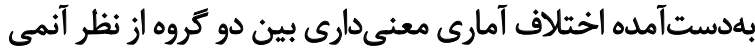

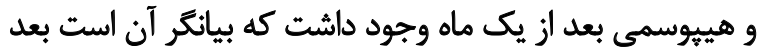

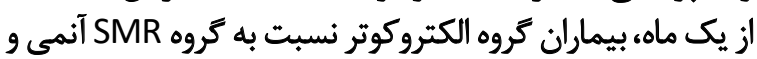

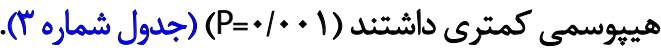

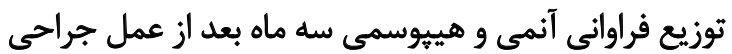

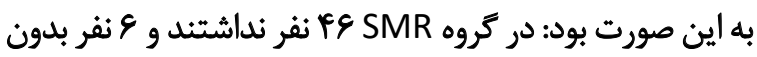

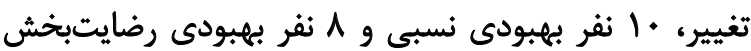

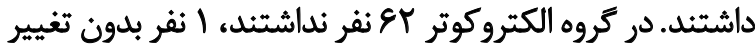

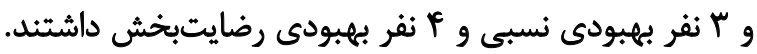

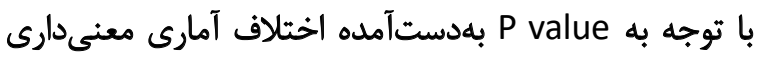

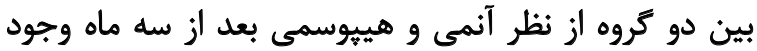

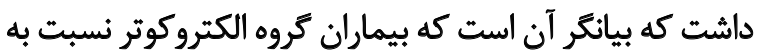

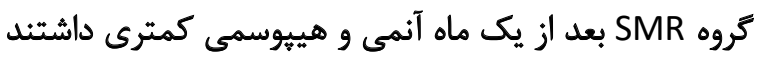

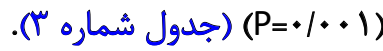

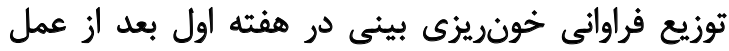

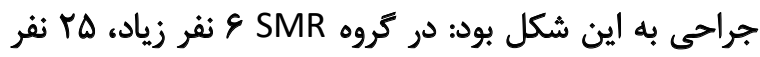

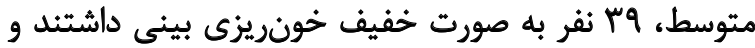

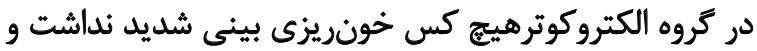

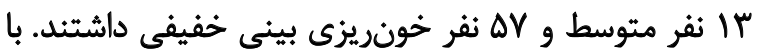

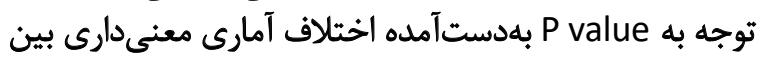

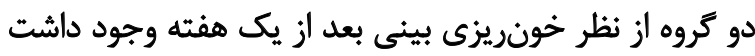

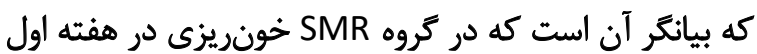

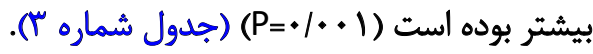

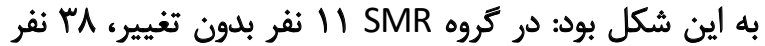

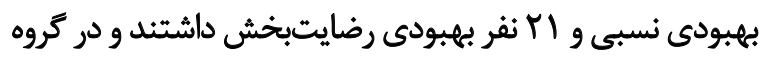

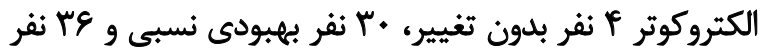

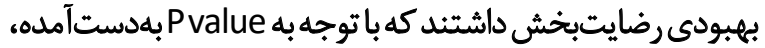

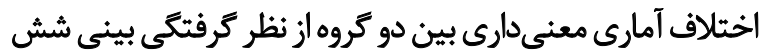

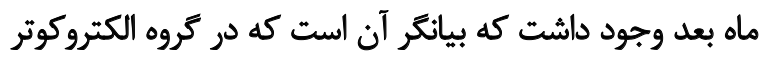

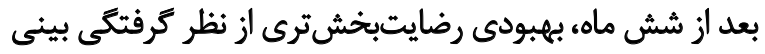

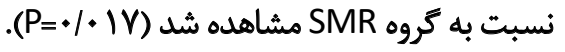

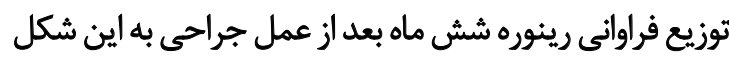

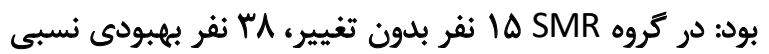

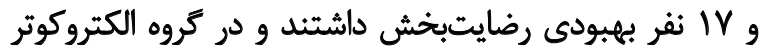

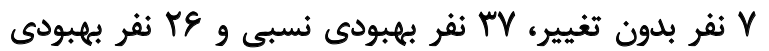

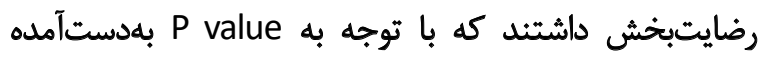

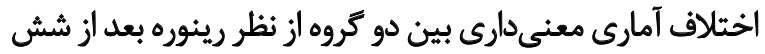

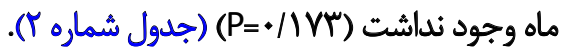

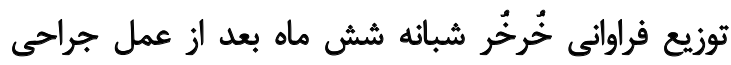

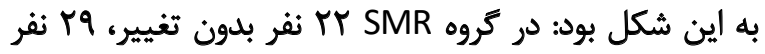

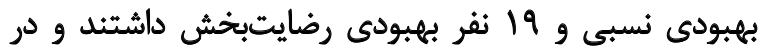

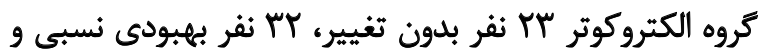

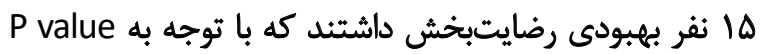

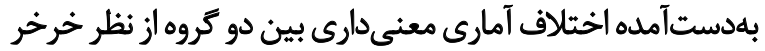

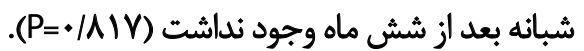
توزيع فراوانى سردرد در ماه اول بعد از عمل جراحى به به اين شكل

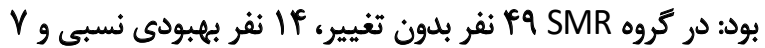

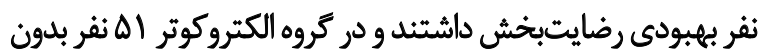

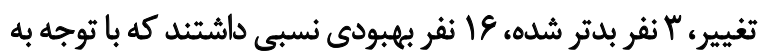

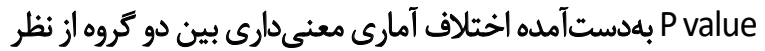

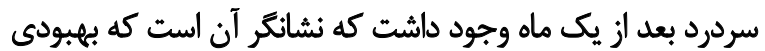

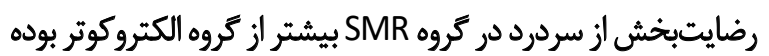

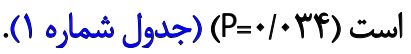

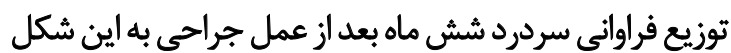

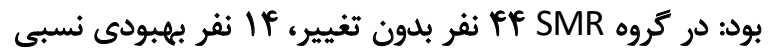

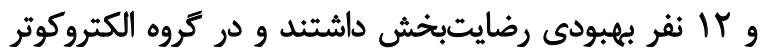

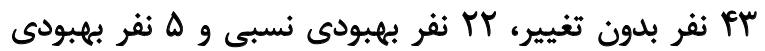

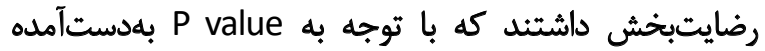

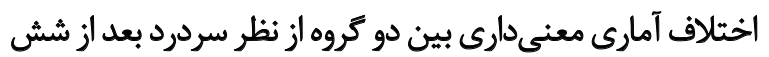
ماه وجود نداشت (P=•//QV) (جدول شماره ل).

توزيع فراوانى درد و سوزش محل جراحي يك ماه بعد از عمل برد برد

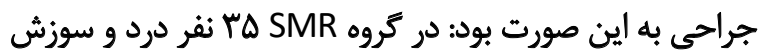

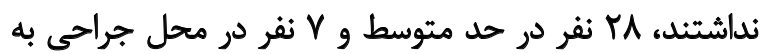

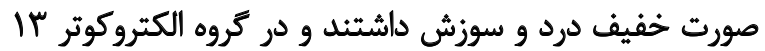

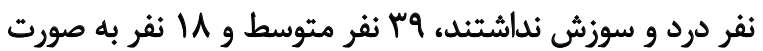


جدول ا. توزيع فراوانى سردرد بعد از عمل جراحى به تفكيك كروه

\begin{tabular}{|c|c|c|c|c|c|}
\hline \multirow{2}{*}{$\mathbf{P}$} & \multirow{2}{*}{ جمع كل } & \multicolumn{2}{|c|}{ كروه } & \multirow{2}{*}{ سردرد } & \\
\hline & & الكتروكوتر & SMR & & \\
\hline \multirow{4}{*}{. $/ 9 \pi r$} & $P Q$ & $M$ & $M$ & بلون تغيير & \multirow{4}{*}{ هفته اول } \\
\hline & 9. & $\Leftrightarrow$ & ri & بدترشلن & \\
\hline & $\Delta$ & $r$ & $r$ & بهبودى نسبي & \\
\hline & + & • & - & بهبودى رضايتبخش & \\
\hline \multirow{4}{*}{$\% m$} & $1+$. & (D) & $p q$ & بلون تغيير & \multirow{4}{*}{ يك ماه بعد } \\
\hline & $r$ & r & • & بدترشلن & \\
\hline & r. & is & if & بهيودى نسبى & \\
\hline & $v$ & - & $\checkmark$ & بهيودى رضايتبخش & \\
\hline \multirow{3}{*}{.$/ 9 \pi r$} & $M$ & prof & pre & بلون تغيير & \multirow{3}{*}{ كله ماه بعد } \\
\hline & f. & $M$ & 19 & بهيودي نسبي & \\
\hline & ir & $\Delta$ & $\checkmark$ & بهيودى رضايتبخش & \\
\hline \multirow{3}{*}{. / IOY } & AY & Pr & pr & بلون تغيير & \multirow{3}{*}{ شش ماه بعد } \\
\hline & re & rr & if & بهبودى نسبى & \\
\hline & iv & $\Delta$ & ir & بهبودى رضايتبخش & \\
\hline
\end{tabular}

ing

جدول r. توزيع فراواني ريثوره بعد از عمل جراحي به تفكيك كروه

\begin{tabular}{|c|c|c|c|c|c|}
\hline \multirow{2}{*}{$\mathbf{P}$} & \multirow{2}{*}{ جمع كل } & \multicolumn{2}{|c|}{ كروه } & \multirow{2}{*}{ رينوره } & \\
\hline & & الكتروكوثر & SMR & & \\
\hline \multirow{4}{*}{.$/ 1 \mathrm{Vq}$} & iv & 10 & r & بلون تغيير & \multirow{4}{*}{ هفته اول } \\
\hline & $\Delta A$ & $m$ & re & بلدتشلن & \\
\hline & is & r & me & بهيودى نسبى & \\
\hline & - & $\cdot$ & - & بيهبودى رضايتبنش & \\
\hline \multirow{4}{*}{$.11 \cdot 4$} & ra & ir & 10 & بلون تغيير & \multirow{4}{*}{ يك ماه بعد } \\
\hline & v & 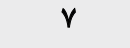 & $\cdot$ & بلدترشن & \\
\hline & $\mathrm{VA}$ & ra & f. & بهيودي نسبي & \\
\hline & tr & ir & 10 & بهبودى رضايتبخش & \\
\hline \multirow{3}{*}{. /rqp } & r & $\wedge$ & 10 & بلون تغيير & \multirow{3}{*}{ سله ماه بعد } \\
\hline & $\mathrm{VA}$ & f. & ra & يهبودى نسبى & \\
\hline & rq & rr & iv & بهيودي رضايتبخش & \\
\hline \multirow{3}{*}{./In } & r & $\checkmark$ & 10 & بلون تغيير & \multirow{3}{*}{ شش ماه بعل } \\
\hline & Va & rv & ऍ & بهبودى نسبي & \\
\hline & rr & re & iv & بهيبودى رضايتبخش & \\
\hline
\end{tabular}


جدول r. توزيع فراوانى خونريزى بينى بعد از عمل جراحى به تفكيك كروه

\begin{tabular}{|c|c|c|c|c|c|}
\hline \multirow{2}{*}{$\mathbf{P}$} & \multirow{2}{*}{ جمع كل } & \multicolumn{2}{|c|}{ كروه } & \multirow{2}{*}{\multicolumn{2}{|c|}{ خونريزى بينى بعد از عمل جراحى }} \\
\hline & & الكتروكوتر & SMR & & \\
\hline \multirow{3}{*}{$.1 .+1$} & er & - & 8 & زياد & \multirow{3}{*}{ 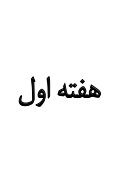 } \\
\hline & rA & זו & ra & متوسط & \\
\hline & 19 & $\Delta V$ & $\mathrm{mq}$ & خفيف & \\
\hline \multirow{2}{*}{ 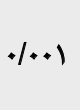 } & $M \mathrm{M}$ & ov & Q) & نلائشته & \multirow{2}{*}{ يك ماه بعد } \\
\hline & tr & r & 19 & خفيف & \\
\hline \multirow{2}{*}{$1 / \ldots$} & itr & $8 \lambda$ & $9 q$ & نلائشته & \multirow[b]{2}{*}{ سه ماه بعد } \\
\hline & $r$ & $r$ & 1 & خفيف & \\
\hline
\end{tabular}

توزيع فراوانى دلمه شش ماه بعد از عمل جراحى به ائ اين صورت

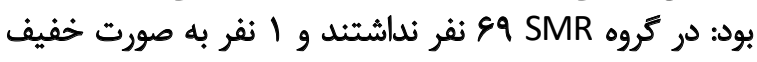

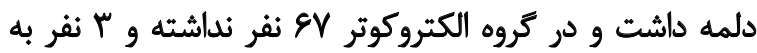

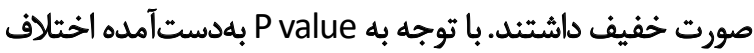

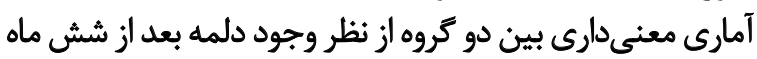

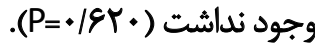

ث

اين مطالعه با هدف مقايسه اثربخشى روش سابموكوزيرال

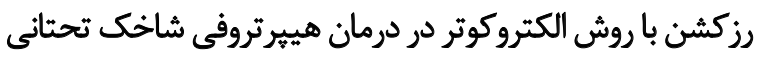

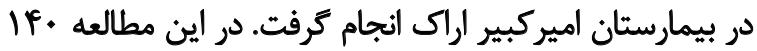

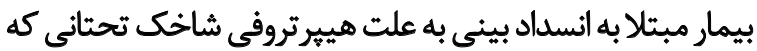

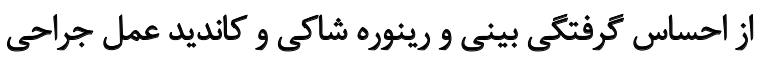

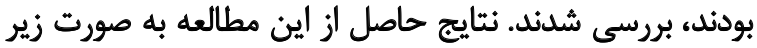

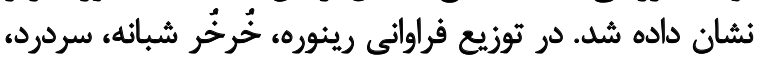

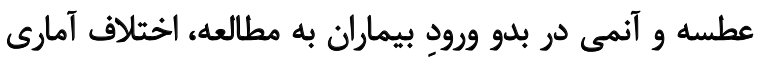

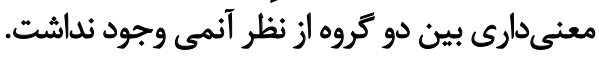

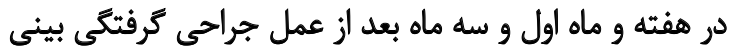

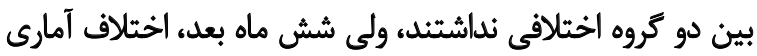

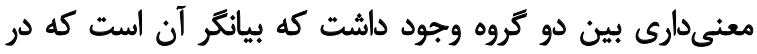

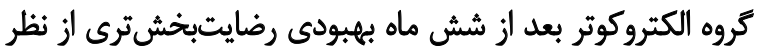

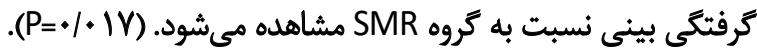

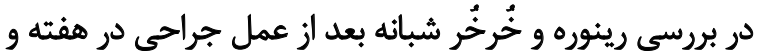

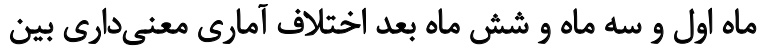

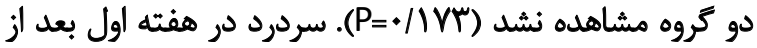

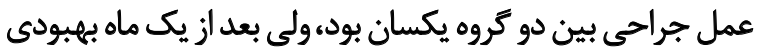

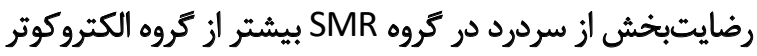

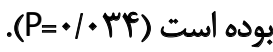

درد و سوزش محل جراحى در هفته اول بعد از عمل جراحى

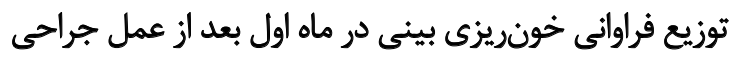

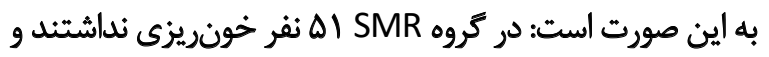
19 نفر خونريزى خفيف داشتند و در ترون

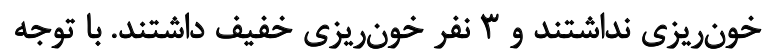

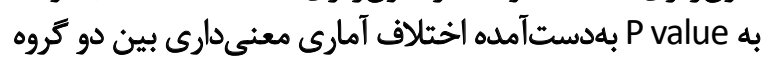

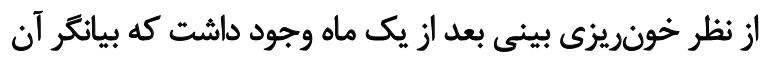

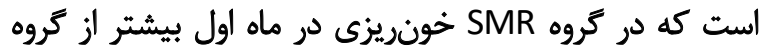

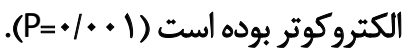

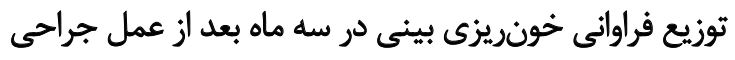

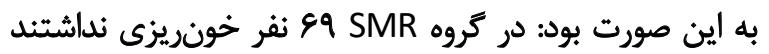

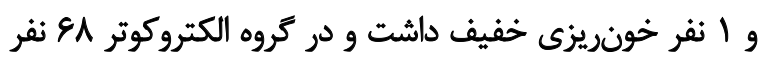

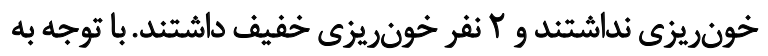

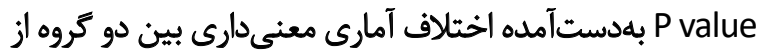

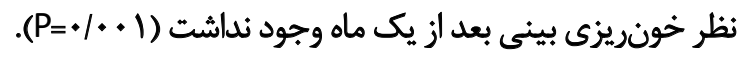
توزيع فراوانى دلمه در هفته اول بعد از عمل جرات براحي به اين

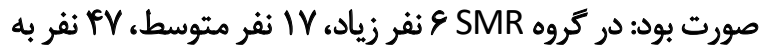

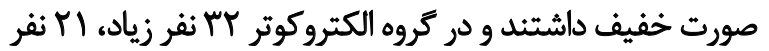

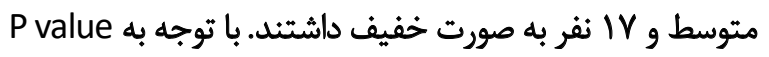

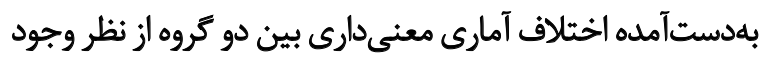

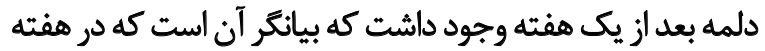

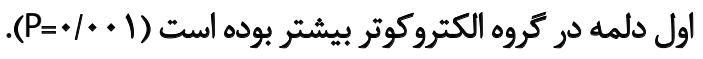

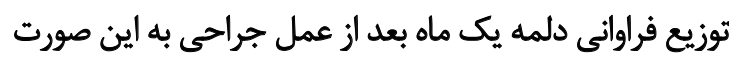

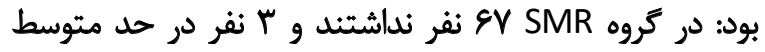

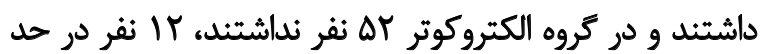

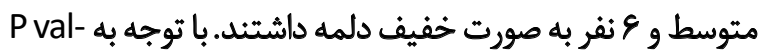

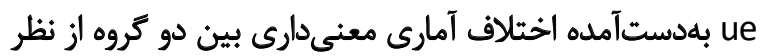

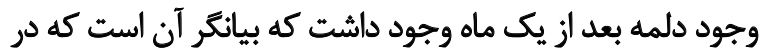

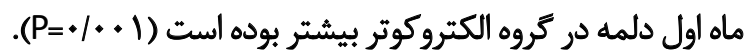




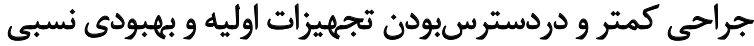

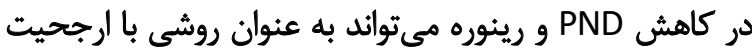

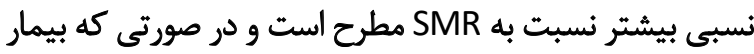

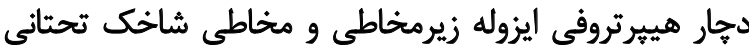

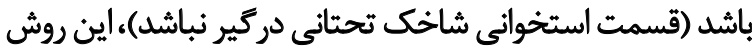

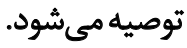

مالاحظات اخلاقى

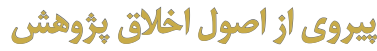

اين ئزوهش را كميته اخلاق دانشعاه علوم يزشكى اراك

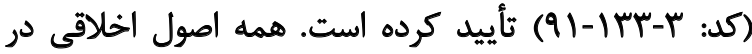

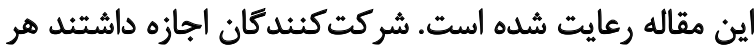

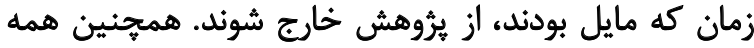

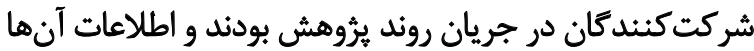

محرمائه نكله داشته شد شند.

$$
\text { Ito solo }
$$

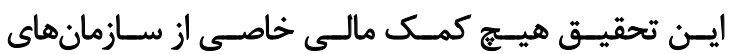

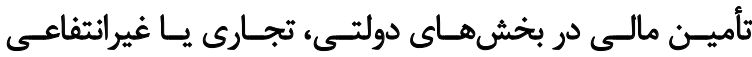

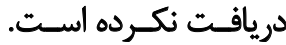

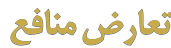

بنابر اظهار نويسنده، اين مقاله تعارض مثافع ندارد.
در هر دو كروه يكسان بود، در حالى كه بعد از يك ماه بيماران

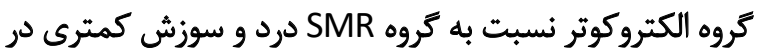

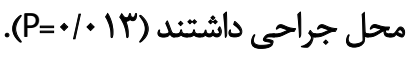

توزيع فراوانى عطسه، سرفه وخارش در هفته اول بعد از عمل

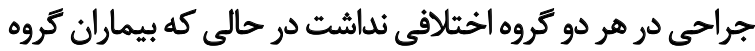

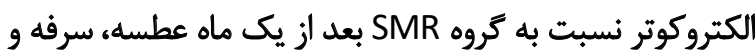

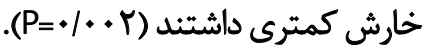

همجئين اختلاف آمارى معنى دارى بين دو كروه از نظر آنمى

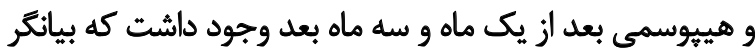

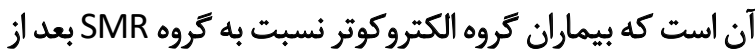

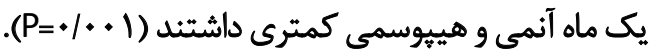

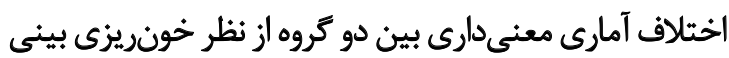

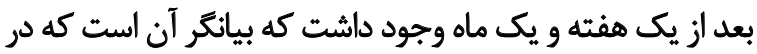

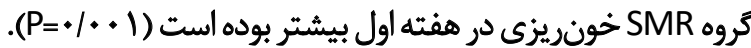

اختلاف آمارى معنى دارى بين دو كروه از نظر وجود دلمه يك ديك

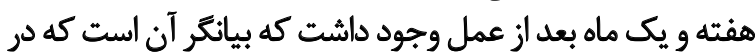

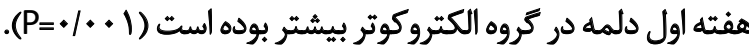
در مطالعه هاسالى" روش SMR با تأثير مطلوب دراز مدت از

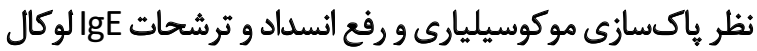

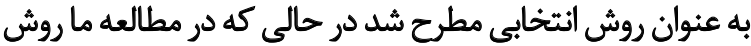

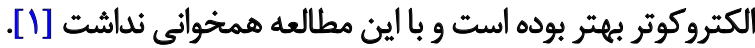

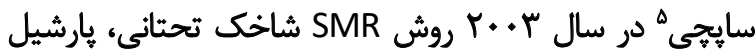

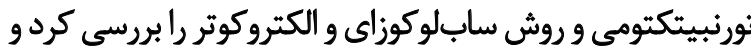
روش SMR رأأييد كرد كه با نتايج مطالعه ما همسو نبوديود.

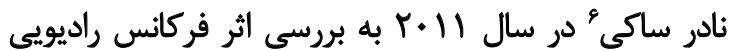

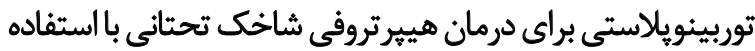

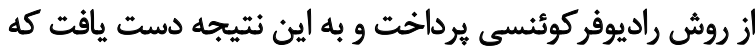

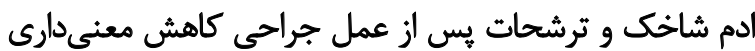

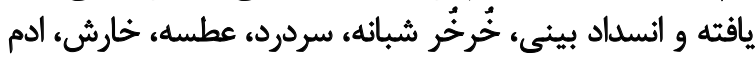

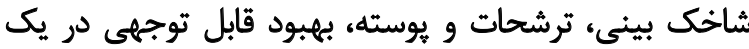

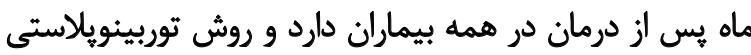

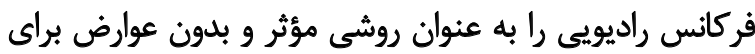

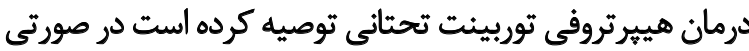

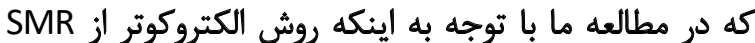

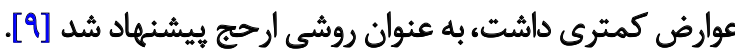

$$
\text { تثيجدكيرى }
$$

روش الكتروكوتر با توجه به خونريزى كمتر و زمان عمل 


\section{References}

[1] Passali D, Passàli FM, Damiani V, Passàli GC, Bellussi L. Treatment of inferior turbinate hypertrophy: A randomized clinical trial. Ann Otol Rhinol Laryngol. 2003; 112(8):682-8. [DOI:10.1177/0003489403112 00806] [PMID]

[2] Leongse SC, Eccles R. Inferior turbinate surgery and nasal airflow: Evidence-based management. Curr Opin Otolaryngol Head Neck Surg. 2010; 18(1):54-9. [DIO:10.1097/MOO.0b013e328334db14] [PMID]

[3] Chen YL, Liu CM, Huang HM. Comparison of microdebrider assisted inferior turbinoplasty and submucosal resection for children with hypertrophic inferior tarbinates. Int J Pediatr Otorhinolaryngol. 2007; 71(6):921-7. [DOI:10.1016/j.ijporl.2007.03.002] [PMID]

[4] Haruhiko I, Yoshida T, Hasegawa T, Mohri M, Amatsu M. Submucous electrocautery following submucous resecrion of turbinate bone-a rationale of surgical treatment for allergic rhinitis. Auris Nasus Larynx. 2003; 30(2):147-52. [DOI:10.1016/S0385-8146(03)00010-5]

[5] Cumming cw, Flint PW, Harker LA, Haughey BH, Richardson MA, Robbins KT. Cummings otolorgrragely Heael and Neck surgery ; $4^{\text {th }}$ ed. Philadelphia: Mosby; 2005.

[6] Salzano FA, Mora R, Dellepiane M, Zannis I, Salzano G, Moran E, et al. Radiofrequency, high-frequency, and electrocautery treatments vs partial inferior turbinotomy: microscopic and macroscopic effects on nasal mucosa. Arch Otolaryngol Head Neck Surg. 2009; 135(8):752-8. [DOI:10.1001/archoto.2009.87] [PMID]

[7] Sapci T, Sahin B, Karavus A, Akbulut UG. Comparison of the effects of radiofrequency tissue ablation, $\mathrm{CO} 2$ laser ablation, and partial turbinectomy applications on nasal mucociliary functions. Laryngoscope. 2003; 113(3):514-9. [DOI:10.1097/00005537-200303000-00022] [PMID]

[8] Bhandarkar ND, Smith TL. Outcomes of surgery for inferior turbinate hypertrophy. Curr Opin Otolaryngol Head Neck Surg. 2010; 18(1):49-53. [DOI:10.1097/MOO.0b013e328334d974] [PMID]

[9] Saki N, Nik Akhlagh S, Hekmat-Shoar M, Saleh Jafari N. Efficacy of radiofrequency turbinatoplasty for treatment of inferior turbinate hypertrophy. Iran J Otorhinolaryngol. 2011; 23(64):87-92.

[10] Cummings CW, Flint PW, Harker LA, Haughey BH, Richardson MA, Robbins KT. Cummings otolaryngology, head and neck surgery. $5^{\text {th }}$ ed. Philadelphia: Mosby; 2010.

[11] Jackson LE, Koch RJ. Controversies in the management of inferior turbinate hypertrophy: A comprehensive review. Plast Reconstr Surg. 1999; 103(1):300-12. [DOI:10.1097/00006534-199901000-00049] [PMID]

[12] Courtiss EH, Goldwyn RM. The effects of nasal surgery on airflow. Plast Reconstr Surg. 1983; 72(1):9-21. [DOI:10.1097/00006534-19830700000003] [PMID] 